\title{
含叔醇结构的新型吡唑-4-甲酰胺类化合物的合成及杀菌活性
}

\author{
耿瑞赵宇李益豪刘金硅砳王明安* \\ (中国农业大学应用化学系 北京 100193)
}

\begin{abstract}
摘要 在吡唑环的 3 位引入叔醇结构, 设计合成了一系列新颖的吡唑-4-甲酰胺类化合物, 其结构经 ${ }^{1} \mathrm{H} N \mathrm{NR},{ }^{13} \mathrm{C} N M R$, HR-ESI-MS 和 X 射线衍射表征确证. 生物活性测试结果显示, 部分化合物在 $100 \mu \mathrm{g} / \mathrm{mL}$ 时对瓜果腐霉菌和辣椒疫霉菌 等测试植物病原菌显示了一定的杀菌活性, 如 $N$-(2-氟苯基)-3-(1-羟基环己基)-1,5-二甲基- $1 H$-吡唑-4-甲酰胺( $\mathbf{B}_{1}$ )和 $N$-(噻唑-2-基)-3-(1-羊基环己基)-1,5-二甲基- $1 H$-吡唑-4-甲酰胺( $\mathbf{B}_{10}$ )对瓜果腐霉菌的 $\mathrm{EC}_{50}$ 分别为 76.3 和 $71.9 \mu \mathrm{g} / \mathrm{mL}$, 化 合物 $\mathbf{B}_{10}$ 对辣椒疫霉菌的 $\mathrm{EC}_{50}$ 分别为 $85.4 \mu \mathrm{g} / \mathrm{mL}$.
\end{abstract}

关键词 吡唑-4-甲酰胺; 叔醇; 合成; 杀菌活性

\section{Synthesis and Fungicidal Activity of Novel Pyrazole-4-carbox- amide Compounds Containing Tertiary Alcohol Moiety}

\author{
Geng, Rui Zhao, Yu Li, Yihao Liu, Xinlei Wang, Ming'an* \\ (Department of Applied Chemistry, China Agricultural University, Beijing 100193)
}

\begin{abstract}
A series of novel pyrazole-4-carboxamide compounds were designed and synthesized through introducing a tertiary alcohol moiety into the $\mathrm{C}-3$ position of the pyrazole ring based on the chracteristics of pyrazole-4-carboxamide fungicides and natural products with a tertiary alcohol moiety. Their structures were characterized by ${ }^{1} \mathrm{H} N M R,{ }^{13} \mathrm{C}$ NMR, HR-ESI-MS data and X-ray diffraction. The preliminary bioassay results indicated that some of these compounds exhibit certain fungicidal activities against tested phytopathogens at the concentration of $100 \mu \mathrm{g} / \mathrm{mL} . N$-(2-Fluorophenyl)-3-(1-hydroxycyclohexyl)-1,5dimethyl-1H-pyrazole-4-carboxamide $\left(\mathbf{B}_{1}\right)$ and $N$-(thiazol-2-yl)-3-(1-hydroxycyclohexyl)-1,5-dimethyl-1 $H$-pyrazole-4-carboxamide $\left(\mathbf{B}_{\mathbf{1 0}}\right)$ have $\mathrm{EC}_{50}$ values of 76.3 and $71.9 \mu \mathrm{g} / \mathrm{mL}$ against Pythium aphanidermatum. $\mathbf{B}_{\mathbf{1 0}}$ has an $\mathrm{EC}_{50}$ value of 85.4 $\mu \mathrm{g} / \mathrm{mL}$ against Phytophthora capsici, respectively.
\end{abstract}

Keywords pyrazole-4-carboxamide; tertiary alcohol moiety; synthesis; fungicidal activity

近年来开发应用的农用杀菌剂中, 酰胺类杀菌剂独 树一帜, 其商品化药物上市数量较多, 同时具备靶标明 确和环境友好等特点, 其中吡唑-4-甲酰胺类杀菌剂成 为国内外研究的热点之一 ${ }^{[1 ~ 9]}$, 目前已商品化的吡唑-4甲酰胺类杀菌剂如氟唑菌酰胺(fluxapyroxad)、联苯吡菌 胺 (bixafen)、氟唑环菌胺(sedaxane)、氟唑菌酰羟胺 (pydiflumetofen)和氟唑菌苯胺(penflufen)等(图 1)已成为 一类重要的农用杀菌剂并获得广泛应用. 叔醇结构是广

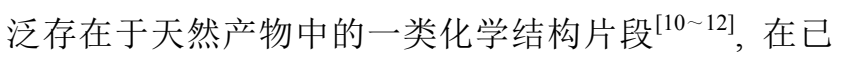
开发的农药或者医药中也经常出现 ${ }^{[13 \sim 15]}$ (图 2), 但是迄 今在吡唑-4-甲酰胺类杀菌剂的研究中尚未见引入叔醇 结构的报道. 我们课题组 ${ }^{[16]}$ 发现经由烯醇醚修饰的丁
烯内酯与胺类反应可以制备两类不同的烯胺酮类化合 物, 而这些丁烯内酯与肼类化合物反应同样可以生成两 类不同的肼基衍生物, 这些肼基衍生物表现出较优异的 农用杀虫和杀菌活性，但是当丁烯内酯与水合肼和甲基 肼反应时意外获得一类未见文献报道的含叔醇结构片 段的吡唑-4-甲酸酯类化合物 ${ }^{[17]}$, 该类吡唑-4-甲酸酯化 合物在吡唑环的 3 位含有叔醇片段, 具有提高吡唑-4-甲 酰胺类化合物杀菌活性的潜能. 因此本工作以该类吡 唑-4-甲酸酯为基础, 将文献报道的具有优异杀菌活性 的吡唑-4-甲酰胺类杀菌剂的胺类基团引入分子中，设 计合成一类新型吡唑-4-甲酰胺类化合物(Eq. 1), 并通过 杀菌活性的评价探索这类新型吡唑-4-甲酰胺是否具有

* Corresponding author. E-mail: wangma@cau.edu.cn

Received June 17, 2019; revised July 8, 2019; published online August 1, 2019.

Project supported by the National Natural Science Foundation of China (No. 21772229).

国家自然科学基金(No. 21772229)资助项目. 
成为新的先导化合物的可能性. 化合物的合成路线如 Scheme 1 所示.<smiles>Cn1cc(C(=O)Nc2ccccc2-c2cc(F)c(F)c(F)c2)c(C(F)F)n1</smiles>
fluxapyroxad<smiles>Cn1cc(C(=O)Nc2ccccc2C2CC2C2CC2)c(C(F)F)n1</smiles>

sedaxane
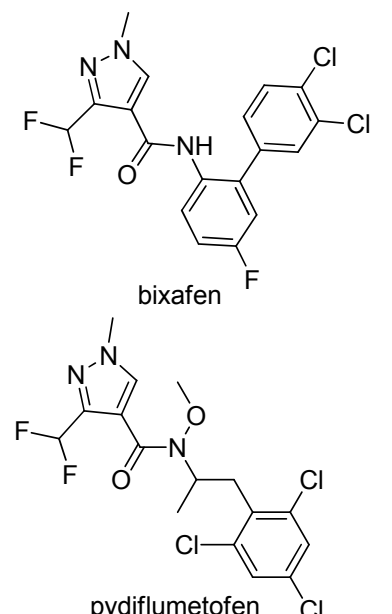<smiles>Cc1nn(C)c(F)c1C(=O)Nc1ccccc1C(C)CC(C)C</smiles>

图 1 典型的吡唑-4-甲酰胺类杀菌剂的化学结构

Figure 1 Structures of typical pyrazole-4-carboxamide fungicides<smiles>COc1ccc(C(O)(c2cncnc2)C2CC2)cc1</smiles>

图 2 典型的含叔醇结构的天然产物和杀菌剂的化学结构

Figure 2 Structures of typical natural products and fungicides with a tertiary alcohol moiety
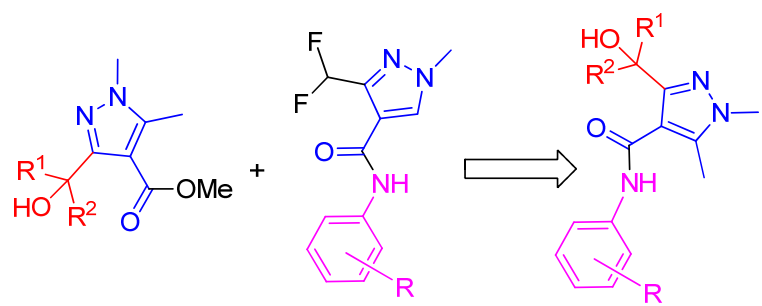

\section{1 结果与讨论}

参照前文 ${ }^{[17]}$ 在合成该系列化合物的过程中开始尝 试使用甲基肼对原料进行亲核进攻，以期直接获得中间<smiles>[R]C([R])(O)c1n[nH]c(C)c1C(=O)OC</smiles>

I-1, I-2<smiles>[R]C([R])(O)c1nn(C)c(C)c1C(=O)O</smiles>

$\mathrm{I}-5, \mathrm{I}-6$<smiles>[R]NC(=O)c1c(C([R])([R])[Y11]#[W])nn(C)c1C</smiles>

A1 A11, B1 B11

I-1, I-3, I-5, I-9, A: $\mathrm{R}^{1}=\mathrm{R}^{2}=\mathrm{CH}_{3}$

I-2, I-4, I-6, I-10, B: $\mathrm{R}^{1} \mathrm{R}^{2}=\left(\mathrm{CH}_{2}\right)_{5}$<smiles>Cc1c2c(nn1C)C(C)(C)OC2=O</smiles><smiles>Cc1c2c(nn1C)C1(CCCCC1)OC2=O</smiles>

图式 1 新型含叔醇结构片段的吡唑-4-甲酰胺类化合物的合 成路线

Scheme 1 Synthetic route of novel pyrazole-4-carboxamide compounds with a tertiary alcohol moiety

体 3-(2-着基丙-2-基)-1,5-二甲基- $1 H$-吡唑-4-甲酸甲酯 I-3. 但是在放大量反应后通过 ${ }^{1} \mathrm{H} N \mathrm{NR}$ 分析发现体系由 I-3 及其同分异构体组成(Scheme 2), 且这两种化合物极 性相同难以分离, 尽管进行了溶剂以及浓度等条件的改 变, 仍难以获得纯的化合物. 当使用水合肼对原料进行 亲核进攻时只得到单一异构体 I-1, 且其结构经 X 射线

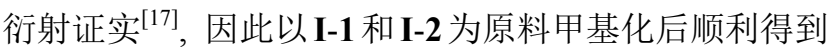
了中间体 I-3 和 I-4. 当得到中间体 I-3 后，发现 I-3 的 ${ }^{1} \mathrm{H}$ NMR 与文献[17]得到的 3-(2-羟基丙-2-基)-1,5-二甲基$1 H$-吡唑-4-甲酸甲酯的 ${ }^{1} \mathrm{H}$ NMR 并不相同(图 3), 因此可 以推定文献得到的化合物是 5-(2-差基丙-2-基)-1,3-二甲 基-1H-吡唑-4-甲酸甲酯，即 I-3 的同分异构体. 中间体 I-3 和 I-4 经水解得到中间体 1,5-二甲基- $1 H$-吡唑-4-甲酸 I-5 和 I-6.<smiles>COC(=O)c1c(I)nn(C)c1C(C)(C)O</smiles>

图式 2 烯醇醚与甲基肼反应产生两种吡唑-4-甲酸甲酯及内 酯化副产物的结构

Scheme 2 Two methyl pyrazole-4-carboxylates from enol ether with methyl hydrazine and structures of byproducts 


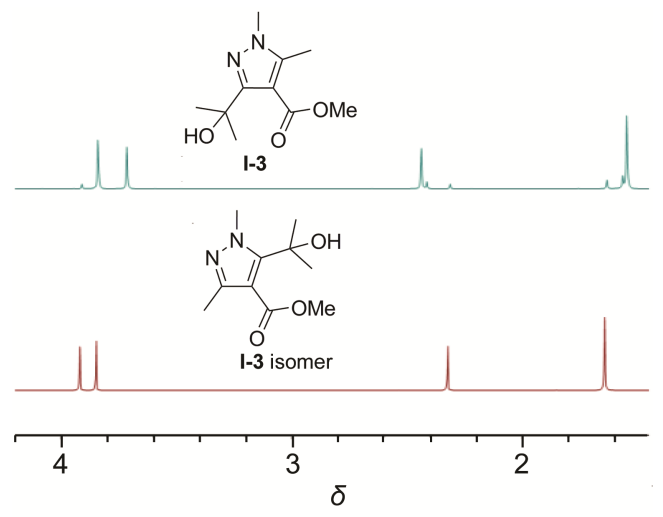

图 3 中间体 I-3 与其异构体的 ${ }^{1} \mathrm{H}$ NMR 比较 Figure $3{ }^{1} \mathrm{H}$ NMR comparison of intermediate I-3 and its isomer

另一方面，由于中间体 I-5 和 I-6 分子中含有叔醇， 直接活化羧基成为酰卤或者酸䣶制备酰胺的方法并不 适用，尝试通过芐醚、四氢吡喃(THP)和叔丁基二甲基氯 硅烷(TBSCl)等保护基对 3 位叔醇进行保护, 均未收到 良好的效果. 1-羟基苯并三唑( $\mathrm{HOBt}$ )活化羧基是制备羧 酸酯和酰胺的常用方法, 为此进行了 HOBt 活化羧基的 尝试, 发现 HOBt 与中间体 I-5 或 I-6 反应后生成两个化 合物, 经过反复实验后确定通过乙酸乙酯重结晶处理可 以以 $50 \% \sim 60 \%$ 的收率获得纯的活化酯 I-9 或 I-10, 母 液通过柱层析纯化可以得到内酯化副产物 I-7 和 I-8, 其 后经强碱水解回收中间体 I-5 和 I-6 而得以重复使用 (Scheme 2). 但是将副产物 I-7 和 I-8 直接与各种胺类反 应制备酰胺的尝试未能成功, 可能与副产物 I-7 和 I-8 具有很好的稳定性有关.

在合成目标产物的过程中, 中间体活化酯 I-9 或 I-10 与苠胺和脂肪胺类的合成反应经薄层色谱(TLC)监 测, 反应体系较为单一且收率较高 $(\mathbf{A 4} \sim \mathrm{A9}, \mathrm{B} 4 \sim \mathbf{B} 9)$, 无明显副产物生成. 但是中间体活化酯 I-9 或 I-10 与杂 环胺和取代苯胺的反应由于反应活性差还会有内酯化 副产物 I-7 和 I-8 生成, 对杂环胺生成的酰胺而言，与内 酯化副产物的极性相差较大通过柱层析易于分离 $(\mathrm{A} 10 \sim \mathrm{A} 11 ， \mathrm{~B} 10 \sim \mathrm{B} 11)$, 而对取代苯胺生成的酰胺而 言, 与内酯化副产物的极性相差较小, 需要进一步将胺 基用 Boc 保护，再进行柱层析分离纯化并脱去保护基才 能得到目标化合物 $(\mathbf{A 1} \sim \mathbf{A 3}, \mathbf{B 1} \sim \mathbf{B} 3)$, 所以他们的收率 较低. 为了证明化合物的化学结构和叔醇的位置, 选择 代表性的化合物 A7 进行了晶体 X 射线衍射, 其结果如 图 4 所示. 该结构证明了吡唑-4-甲酰胺的成功合成和叔 醇片段在吡唑环的 3 位, 与前述 ${ }^{1} \mathrm{H}$ NMR 分析的结论一 致，同时显示叔醇的羟基氧原子与酰胺键的氮和氢原子 间形成较稳定的七元环氢键, 进一步了稳定该类化合物 的结构, 这与前文 3-(2-羟基丙- 2-基)-5-甲基-1H-吡唑-
4-甲酸甲酯 I-1 的晶体结构中叔醇的羟基与羧基的羰基 氧原子形成的七元环氢键情况相似 ${ }^{[17]}$.

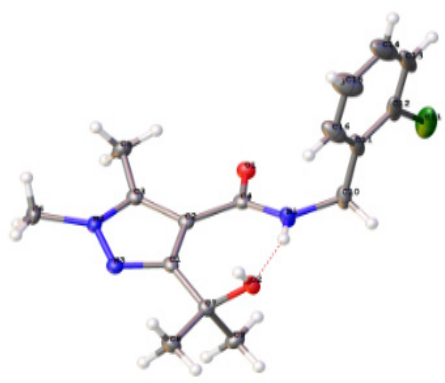

图 4 化合物 $\mathrm{A} 7$ 的晶体结构

Figure 4 Crystal structure of compound A7

采用菌丝生长速率法 ${ }^{[18-20]}$ 对目标化合物的杀菌活性 进行了评价, 结果如表 1 所示. 结果显示在 $100 \mu \mathrm{g} / \mathrm{mL}$ 的 浓度下，所有目标化合物对测试的六种植物病原菌都显 示出一定的离体杀菌活性，抑制率均小于 $70 \%$, 不如对 照药剂醚菌酯和啶酰菌胺的杀菌活性，但是化合物 B1 和 B10 对瓜果腐霉菌的抑制率仍达到 $67.8 \%$ 和 $69.0 \%$, B10 对辣椒疫霉菌的抑制率达到 $62.4 \%$. 通过对比发现 酰胺键所连基团为噻唑胺等杂环胺时, 化合物的杀菌活 性显著提高，说明该类化合物引入杂环胺可以改善生物 活性，整体来看，胺类基团对于杀菌活性的影响顺序是： 含氮杂环胺类 $>$ 取代苯胺类 $>$ 芐胺类 $=$ 脂肪胺类; 另一 方面，含有 3 位偕二甲基叔醇的 $\mathbf{A}$ 类化合物的杀菌活性 比 3 位环己基叔醇的 $\mathbf{B}$ 类化合物的杀菌活性稍差，表明 3 位具有一定空间位阻的叔醇结构可以提高该类化合物 的杀菌活性. 在此基础上, 进一步测试了化合物 B1 和 B10 对瓜果腐霉菌的半有效抑制浓度 $\left(\mathrm{EC}_{50}\right)$ 值, 分别为 76.3 和 $71.9 \mu \mathrm{g} / \mathrm{mL}$, 显著高于啶酰菌胺 $\mathrm{EC}_{50}$ 的 22.8 $\mu \mathrm{g} / \mathrm{mL}$, 化合物 $\mathbf{B 1 0}$ 对辣椒疫霉菌的 $\mathrm{EC}_{50}$ 值为 85.4 $\mu \mathrm{g} / \mathrm{mL}$ ，也显著高于啶酰菌胺 $\mathrm{EC}_{50}$ 的 $48.1 \mu \mathrm{g} / \mathrm{mL}$. 与其 他商品化的吡唑-4-甲酰胺类杀菌剂相比，该类化合物 的杀菌活性明显降低, 结构差异在于 3 位叔醇和 5 位的 质子或卤素, 说明 3 位和 5 位基团对活性影响很大，希 望可以通过将该类化合物 5 位甲基改造为二氟甲基或三 氟甲基来提高其杀菌活性，有关的结构改造研究工作正 在进行之中.

\section{2 结论}

根据吡唑-4-甲酰胺类杀菌剂的结构特征以及天然 产物和一些农用杀菌剂中含有叔醇结构片段的特点, 在 吡唑环的 3 位引入叔醇结构, 设计合成了一系列新颖的 吡唑-4-甲酰胺类化合物，他们的结构经过 ${ }^{1} \mathrm{H}$ NMR, ${ }^{13} \mathrm{C}$ NMR, HR-ESI-MS 和 X 射线衍射的确证. 生物活性测试 
表 $1100 \mu \mathrm{g} / \mathrm{mL}$ 浓度下化合物 $\mathbf{A 1} \sim \mathbf{A 1 1}, \mathbf{B 1} \sim \mathbf{B 1 1}$ 的离体杀菌活性(抑制率/\%)

Table 1 In vitro fungicidal activities of compounds A1 $\sim \mathbf{A 1 1}, \mathbf{B 1} \sim \mathbf{B} 11$ at $100 \mu \mathrm{g} / \mathrm{mL}$ (inhibition rate $/ \%$ )

\begin{tabular}{|c|c|c|c|c|c|c|c|}
\hline Compd. & $\mathrm{R}^{3}$ & 小麦赤霉菌 & 瓜果腐霉菌 & 辣椒疫霉菌 & 番茄灰霉菌 & 水稻纹枯菌 & 水稻疫霉菌 \\
\hline A1 & $2-\mathrm{FC}_{6} \mathrm{H}_{4}$ & 35.0 & 4.7 & 11.2 & 22.6 & 4.7 & 29.3 \\
\hline A2 & $4-\mathrm{FC}_{6} \mathrm{H}_{4}$ & 30.8 & 14.1 & 8.7 & 11.5 & 7.1 & 16.6 \\
\hline $\mathbf{A 3}$ & $4-\mathrm{CF}_{3} \mathrm{C}_{6} \mathrm{H}_{4}$ & 31.2 & 11.6 & 16.8 & 11.9 & 4.9 & 19.0 \\
\hline A4 & $\mathrm{C}_{6} \mathrm{H}_{5} \mathrm{CH}_{2}$ & 23.3 & 3.0 & 7.9 & 8.3 & 14.4 & 14.3 \\
\hline A5 & $\mathrm{C}_{6} \mathrm{H}_{5} \mathrm{CH}\left(\mathrm{CH}_{3}\right)$ & 36.2 & 8.9 & 17.8 & 26.1 & 24.2 & 11.1 \\
\hline A6 & $4-t-\mathrm{BuC}_{6} \mathrm{H}_{4} \mathrm{CH}_{2}$ & 27.5 & 7.0 & 10.2 & 8.7 & 12.3 & 13.5 \\
\hline A7 & $2-\mathrm{ClC}_{6} \mathrm{H}_{4} \mathrm{CH}_{2}$ & 27.5 & 18.2 & 10.9 & 16.6 & 26.0 & 38.9 \\
\hline A8 & $4-\mathrm{FC}_{6} \mathrm{H}_{4} \mathrm{CH}_{2}$ & 7.1 & 57.1 & 3.3 & 12.1 & 8.3 & 3.7 \\
\hline A9 & $t$-Butyl & 13.3 & 11.2 & 15.9 & 26.1 & 25.2 & 11.9 \\
\hline A10 & Thiazol-2-yl & 37.5 & 5.8 & 39.5 & 8.7 & 52.3 & 19.8 \\
\hline A11 & Thiadiazol-2-yl & 42.5 & 19.2 & 15.7 & 7.1 & 33.3 & 16.7 \\
\hline B1 & $2-\mathrm{FC}_{6} \mathrm{H}_{4}$ & 29.7 & 67.8 & 13.4 & 31.7 & 21.4 & 15.1 \\
\hline B2 & $4-\mathrm{FC}_{6} \mathrm{H}_{4}$ & 41.2 & 24.6 & 14.3 & 19.0 & 10.2 & 23.6 \\
\hline B3 & 4- $\mathrm{CF}_{3} \mathrm{C}_{6} \mathrm{H}_{4}$ & 21.4 & 16.2 & 4.7 & 19.8 & 9.7 & 11.8 \\
\hline B4 & $\mathrm{C}_{6} \mathrm{H}_{5} \mathrm{CH}_{2}$ & 27.3 & 5.8 & 10.5 & 25.4 & 15.0 & 10.9 \\
\hline B5 & $\mathrm{C}_{6} \mathrm{H}_{5} \mathrm{CH}\left(\mathrm{CH}_{3}\right)$ & 18.2 & 18.2 & 8.3 & 25.0 & 4.6 & 5.2 \\
\hline B6 & $4-t-\mathrm{BuC}_{6} \mathrm{H}_{4} \mathrm{CH}_{2}$ & 14.2 & 7.8 & 7.1 & 14.2 & 7.1 & 7.5 \\
\hline B7 & $2-\mathrm{ClC}_{6} \mathrm{H}_{4} \mathrm{CH}_{2}$ & 20.8 & 33.2 & 16.3 & 10.3 & 11.7 & 14.4 \\
\hline B8 & $4-\mathrm{FC}_{6} \mathrm{H}_{4} \mathrm{CH}_{2}$ & 11.5 & 29.5 & 10.2 & 9.4 & 13.8 & 16.7 \\
\hline B9 & $t$-Butyl & 22.6 & 10.2 & 5.9 & 4.7 & 11.9 & 11.3 \\
\hline B10 & Thiazol-2-yl & 30.1 & 69.0 & 62.4 & 38.1 & 6.7 & 20.3 \\
\hline B11 & Thiadiazol-2-yl & 28.5 & 35.7 & 23.8 & 4.7 & 19.0 & 16.5 \\
\hline 醚菌酯 & & 73.7 & 100 & 34.5 & 78.5 & 69.0 & 82.3 \\
\hline 啶酰菌胺 & & 97.6 & 100 & 82.0 & 100 & 86.0 & 92.9 \\
\hline
\end{tabular}

结果显示, 部分化合物对瓜果腐霉菌和辣椒疫霉菌等测 试植物病原菌显示了一定的杀菌活性, 化合物 $\mathbf{B}_{\mathbf{1}}$ 和 $\mathbf{B}_{10}$ 对瓜果腐霉菌的 $\mathrm{EC}_{50}$ 分别为 76.3 和 $71.9 \mu \mathrm{g} / \mathrm{mL}$, 化合 物 $\mathbf{B}_{\mathbf{1 0}}$ 对辣椒疫霉菌的 $\mathrm{EC}_{50}$ 分别为 $85.4 \mu \mathrm{g} / \mathrm{mL}$. 初步的 结构-活性关系显示, 杂环胺和具有一定空间位阻的 3 位叔醇结构可以提高该类化合物的杀菌活性.

\section{3 实验部分}

\section{1 仪器与试剂}

WRS-3 型显微熔点仪(温度计未校正); Bruker DPX $300 \mathrm{MHz}$ 核磁共振仪, TMS 为内标, $\mathrm{CDCl}_{3}$ 为溶剂; $\mathrm{Ag}-$ ilent 1100 LC-MSD-Trap 质谱仪(ESI-MS), Thermo Fisher MSQ-Plus 型高分辨质谱仪; Thermo Fisher ESCALAB 250 四圆 $\mathrm{X}$ 射线衍射仪; 有机溶液使用旋转蒸发仪浓 缩. 本实验所用柱层析硅胶为青岛海洋化工有限公司生 产(200 300 目), 所用试剂均为国产或进口分析纯, 无 水溶剂用常规方法干燥处理.

\section{2 化合物的合成}

\subsubsection{5-甲基- $1 H$-吡唑-4-甲酸酯 I-1 和 I-2 的合成}

化合物 I-1 和 I-2 的合成参照前文方法进行, 其熔点 和 ${ }^{1} \mathrm{H}$ NMR 数据与文献[17]一致.
3.2.2 1,5-二甲基- $1 H$-吡唑-4-甲酸酯 $\mathbf{I}-\mathbf{3}$ 和 $\mathbf{I}-\mathbf{4}$ 的合成

在 $250 \mathrm{~mL}$ 三口瓶中加入 5-甲基- $1 H$-吡唑甲酸酯 (I-1) $(5 \mathrm{mmol}, 0.99 \mathrm{~g}), 100 \mathrm{~mL}$ 乙腈和碳酸钾 $(8 \mathrm{mmol}$, $1.104 \mathrm{~g}$ ), 室温搅拌 $3 \mathrm{~h}$, 向体系中缓慢滴加 1.5 equiv. 碘 甲烷 $(8 \mathrm{mmol}, 0.507 \mathrm{~mL})$ 的 $10 \mathrm{~mL}$ 乙腈溶液，室温下搅拌 反应 $8 \mathrm{~h}$. 薄层色谱(TLC)监测反应完毕后, 抽滤除去体 系中的碳酸钾, 滤液转移至分液漏斗, 加入 $30 \mathrm{~mL}$ 饱和 硫代硫酸钠溶液洗脱有机相三次，有机相用无水硫酸钠 干燥, 旋转蒸发除去溶剂. 经硅胶柱层析 $[200 \sim 300$ 目 硅胶, 以石油醚/乙酸乙酯为洗脱剂, $V$ (石油醚) : $V$ (乙酸 乙酯 $)=1: 1$ ]分离, 得到 $1.042 \mathrm{~g}$ 淡黄色固体 I-3, 收率 93\%. m.p. 83 85 ${ }^{\circ} \mathrm{C}$; ${ }^{1} \mathrm{H}$ NMR (300 MHz, $\left.\mathrm{CDCl}_{3}\right) \delta$ : $5.96(\mathrm{~s}, 1 \mathrm{H}, \mathrm{OH}), 3.85\left(\mathrm{~s}, 3 \mathrm{H}, \mathrm{OCH}_{3}\right), 3.77\left(\mathrm{~s}, 3 \mathrm{H}, \mathrm{NCH}_{3}\right)$, $2.44\left(\mathrm{~s}, 3 \mathrm{H}, \mathrm{CH}_{3}\right), 1.55\left(\mathrm{~s}, 6 \mathrm{H}, \mathrm{CH}_{3} \times 2\right)$; HR-ESI-MS cacld for $\mathrm{C}_{10} \mathrm{H}_{17} \mathrm{~N}_{2} \mathrm{O}_{3}[\mathrm{M}+\mathrm{H}]^{+}$213.1234, found 213.1238 .

采用同样的方法制得 $1.134 \mathrm{~g}$ 白色固体 I-4, 收率 90\%. m.p. 73 $75{ }^{\circ} \mathrm{C}$; ${ }^{1} \mathrm{H}$ NMR (300 MHz, $\left.\mathrm{CDCl}_{3}\right) \delta$ : $5.68(\mathrm{~s}, 1 \mathrm{H}, \mathrm{OH}), 3.85\left(\mathrm{~s}, 3 \mathrm{H}, \mathrm{OCH}_{3}\right), 3.74\left(\mathrm{~s}, 3 \mathrm{H}, \mathrm{NCH}_{3}\right)$, $2.45\left(\mathrm{~s}, 3 \mathrm{H}, \mathrm{CH}_{3}\right), 2.15 \sim 1.55\left(\mathrm{~m}, 10 \mathrm{H}, \mathrm{CH}_{2} \times 5\right)$; HR-ESIMS cacld for $\mathrm{C}_{13} \mathrm{H}_{21} \mathrm{~N}_{2} \mathrm{O}_{3}[\mathrm{M}+\mathrm{H}]^{+}$253.1547, found 253.1545 . 


\subsubsection{1,5-二甲基- $1 H$-吡唑-4-甲酸 I-5 和 I-6 的合成}

在 $100 \mathrm{~mL}$ 单口圆底烧瓶中加入吡唑-4-甲酸酯 $\mathbf{I - 3}$ $(2 \mathrm{mmol}, 0.424 \mathrm{~g}), 15 \mathrm{~mL}$ 甲醇以及 $15 \mathrm{~mL}$ 去离子水, 充 分搅拌, 再向体系中加入 3 equiv. 的氢氧化锂 $(6 \mathrm{mmol}$, $0.252 \mathrm{~g}$ ), 升温至 $70^{\circ} \mathrm{C}$, 体系呈浑浊状, 持续搅拌 $3 \mathrm{~h}$ 后, 通过 TLC 监测反应, 对比原料至反应完成. 停止加热, 旋蒸除去甲醇, 剩余的水相以 $1 \mathrm{~mol} / \mathrm{L}$ 浓度的稀盐酸调 节 $\mathrm{pH}$ 到 1 , 以乙酸乙酯萃取 $(30 \mathrm{~mL} \times 3)$, 合并有机相, 加入无水硫酸钠进行干燥. 过滤除去固体, 减压除去溶 剂并干燥, 得到 $0.388 \mathrm{~g}$ 淡黄色固体 I-5, 收率 98\%. m.p. $145 \sim 147{ }^{\circ} \mathrm{C} ;{ }^{1} \mathrm{H}$ NMR $\left(300 \mathrm{MHz}, \mathrm{CDCl}_{3}\right) \delta: 3.77$ (s, 3H, $\left.\mathrm{NCH}_{3}\right), 2.54\left(\mathrm{~s}, 3 \mathrm{H}, \mathrm{CH}_{3}\right), 1.62\left(\mathrm{~s}, 6 \mathrm{H}, \mathrm{CH}_{3} \times 2\right)$; HR-ESIMS cacld for $\mathrm{C}_{9} \mathrm{H}_{15} \mathrm{~N}_{2} \mathrm{O}_{3}[\mathrm{M}+\mathrm{H}]^{+}$199.1077, found 199.1081 .

采用同样的方法制得 $0.452 \mathrm{~g}$ 白色固体 I-6, 收率 95\%. m.p. $148 \sim 150{ }^{\circ} \mathrm{C} ;{ }^{1} \mathrm{H}$ NMR $\left(300 \mathrm{MHz}, \mathrm{CDCl}_{3}\right) \delta$ : $3.76\left(\mathrm{~s}, 3 \mathrm{H}, \mathrm{NCH}_{3}\right), 2.53$ (s, 3H, $\left.\mathrm{CH}_{3}\right), 2.14-1.52(\mathrm{~m}, 10 \mathrm{H}$, $\left.\mathrm{CH}_{2} \times 5\right)$; HR-ESI-MS cacld for $\mathrm{C}_{12} \mathrm{H}_{19} \mathrm{~N}_{2} \mathrm{O}_{3}[\mathrm{M}+\mathrm{H}]^{+}$ 239.1390 , found 239.1386 .

3.2.4 1,5-二甲基- $1 H$-吡唑-4-甲酸活化酯 I-9 和 I-10 的合成

在 $100 \mathrm{~mL}$ 单口圆底烧瓶中加入吡唑-4-甲酸 I-5 (1.87 mmol, $370 \mathrm{mg}$ ) 和 $30 \mathrm{~mL}$ 二氯甲烷, 搅拌至固体溶 解后, 将 1.25 equiv. 的 HOBt (2.34 mmol, $315 \mathrm{mg}$ ) 和 1.25 equiv.的 1-乙基-(3-二甲基氨基丙基)碳酰二亚胺(EDCI) (2.34 mmol, $416 \mathrm{mg}$ )在冰浴条件下加入体系, 反应 30 min 后, 溶液体系颜色由黄色变为淡紫色. 经 TCL 检测, 反应原料消失, 停止反应. 旋蒸除去二氯甲烷, 改用 30 $\mathrm{mL}$ 乙酸乙酯溶解样品并转移至分液漏斗, 加入水 $(30$ $\mathrm{mL} \times 3$ )洗掉体系中过量 $\mathrm{HOBt}$, 有机相经无水硫酸钠干 燥, 除去溶剂后的残余物经乙酸乙酯重结晶获得 312 $\mathrm{mg}$ 白色粉末状固体 I-9, 收率 53\%, 母液经硅胶柱层析 分离得到 $148 \mathrm{mg}$ 白色粉末状固体副产物 I-7, 收率 44\%. m.p. 85 86 ${ }^{\circ} \mathrm{C} ;{ }^{1} \mathrm{H}$ NMR $\left(300 \mathrm{MHz}, \mathrm{CDCl}_{3}\right) \delta: 3.83$ (s, $\left.3 \mathrm{H}, \mathrm{CH}_{3}\right), 2.44\left(\mathrm{~s}, 3 \mathrm{H}, \mathrm{CH}_{3}\right), 1.65\left(\mathrm{~s}, 6 \mathrm{H}, \mathrm{CH}_{3} \times 2\right)$; HR-ESI-MS cacld for $\mathrm{C}_{9} \mathrm{H}_{13} \mathrm{~N}_{2} \mathrm{O}_{2}[\mathrm{M}+\mathrm{H}]^{+}$181.0972, found 181.0978 .

采用同样的方法制得白色粉末状固体 I-10, 收率 $60 \%$. 母液经硅胶柱层析分离得到白色粉末状固体副产 物 I-8, 收率 38\%. m.p. 96 98 ${ }^{\circ} \mathrm{C} ;{ }^{1} \mathrm{H}$ NMR (300 MHz, $\left.\mathrm{CDCl}_{3}\right) \delta: 3.82\left(\mathrm{~s}, 3 \mathrm{H}, \mathrm{CH}_{3}\right), 2.45\left(\mathrm{~s}, 3 \mathrm{H}, \mathrm{CH}_{3}\right), 2.15 \sim 1.50$ (m, $10 \mathrm{H}, \mathrm{CH}_{2} \times 5$ ); HR-ESI-MS cacld for $\mathrm{C}_{12} \mathrm{H}_{17} \mathrm{~N}_{2} \mathrm{O}_{2}$ $[\mathrm{M}+\mathrm{H}]^{+}$221.1285, found 221.1282 .

中间体 I-9 和 I-10 未经进一步纯化直接用于下一步 反应.
3.2.5 1,5-二甲基- $1 H$-吡唑-4-甲酰胺 $\mathbf{A 1} \sim \mathbf{A} 3$ 和 $\mathbf{B} 1 \sim$ B3 的合成

以化合物 $\mathrm{A} 1$ 的合成为例, 将吡唑-4-甲酸活化酯中 间体(I-9, $1.0 \mathrm{mmol}, 315 \mathrm{mg})$, 2-氟苯胺(1.5 mmol, 166 $\mathrm{mg}$ )和 $30 \mathrm{~mL}$ THF 加入 $100 \mathrm{~mL}$ 单口圆底烧瓶中, 用油浴 加热至回流反应 $10 \mathrm{~h}$. TLC 监测反应至完全, 减压除去 溶剂后经硅胶柱层析[200 300 目硅胶, 以石油醚/乙酸 乙酯为洗脱剂, $V$ (石油醚) : $V($ 乙酸乙酯 $)=2: 1$ ] 分离, 得到 $0.19 \mathrm{~g}$ 无色固体. 以二氯甲烷溶解反应生成物并加 入催化量的 4- $N, N$-二甲基氨基吡啶(DMAP) (0.01 mmol, $1 \mathrm{mg}$ ), 在反应体系中加入二-叔丁基二碳酸酯(Boc 酸 酎)(1.1 mmol, $205 \mathrm{mg})$, 在室温下搅拌 $3 \mathrm{~h}$, TLC 监测反 应. 粗产物进行硅胶柱层析 $[200 \sim 300$ 目硅胶, 以石油 醚/乙酸乙酯为洗脱剂, $V$ (石油醚) $: V$ (乙酸乙酯 $)=5: 1]$ 分离得到白色固体. 将白色固体用三氟乙酸(TFA)/二氯 甲烷 $(\mathrm{DCM})[V(\mathrm{TFA}): V(\mathrm{DCM})=1 ： 1]$ 混合溶剂溶解, 常温搅拌 $3 \mathrm{~h}$, 除去溶剂, 残余物经硅胶柱层析[200 300 目硅胶, 以石油醚/乙酸乙酯为洗脱剂, $V$ (石油醚)： $V($ 乙酸乙酯 $)=2: 1$ ]分离, 得到 $122 \mathrm{mg}$ 无色针状晶体 $\mathbf{A 1}$, 收率 43\%. 采用同样的方法制得 $\mathbf{A} 2 \sim \mathbf{A 3}$ 和 $\mathbf{B 1} \sim$ B3.

$N$-(2-氟苯基)-3-(2-羟基丙基-2-基)-1,5-二甲基- $1 H$ 吡唑-4-甲酰胺(A1): 收率 43\%, 无色针状晶体. m.p. $120 \sim 122{ }^{\circ} \mathrm{C}$; ${ }^{1} \mathrm{H}$ NMR (300 MHz, $\left.\mathrm{CDCl}_{3}\right) \delta: 10.49$ (s, $1 \mathrm{H}, \mathrm{NH}), 8.43 \sim 8.38(\mathrm{~m}, 1 \mathrm{H}, \mathrm{ArH}), 7.16 \sim 7.01(\mathrm{~m}, 3 \mathrm{H}$, ArH), 3.73 (s, 3H, $\left.\mathrm{NCH}_{3}\right), 2.55\left(\mathrm{~s}, 3 \mathrm{H}, \mathrm{CH}_{3}\right), 1.68(\mathrm{~s}, 6 \mathrm{H}$, $\left.\mathrm{CH}_{3} \times 2\right) ;{ }^{13} \mathrm{C} \mathrm{NMR}\left(75 \mathrm{MHz}, \mathrm{CDCl}_{3}\right) \delta: 162.88,153.89$, $152.84\left(\mathrm{~d},{ }^{1} J_{\mathrm{FC}}=242.3 \mathrm{~Hz}\right), 144.09,126.68\left(\mathrm{~d},{ }^{3} J_{\mathrm{FC}}=9.8\right.$ $\mathrm{Hz}), 123.84\left(\mathrm{~d},{ }^{2} J_{\mathrm{FC}}=27.8 \mathrm{~Hz}\right), 123.76\left(\mathrm{~d},{ }^{2} J_{\mathrm{FC}}=31.5 \mathrm{~Hz}\right)$, $122.15\left(\mathrm{~d},{ }^{4} J_{\mathrm{FC}}=1.5 \mathrm{~Hz}\right), 114.55\left(\mathrm{~d},{ }^{3} J_{\mathrm{FC}}=19.5 \mathrm{~Hz}\right)$, $112.45,70.67,35.76,29.06,11.07$. HR-ESI-MS cacld for $\mathrm{C}_{15} \mathrm{H}_{19} \mathrm{FN}_{3} \mathrm{O}_{2}[\mathrm{M}-\mathrm{H}]^{-}$290.1310, found 290.1304.

$\mathrm{N}$-(4-氟苯基)-3-(2-羟基丙基-2-基)-1,5-二甲基- $1 H$ 吡唑-4-甲酰胺(A2): 收率 45\%, 无色针状晶体. m.p. 205 207 ${ }^{\circ} \mathrm{C} ;{ }^{1} \mathrm{H}$ NMR (300 MHz, $\left.\mathrm{CDCl}_{3}\right) \delta$ : 11.00 (s, $1 \mathrm{H}, \mathrm{NH}), 7.80 \sim 7.55(\mathrm{~m}, 4 \mathrm{H}, \mathrm{ArH}), 3.75\left(\mathrm{~s}, 3 \mathrm{H}, \mathrm{NCH}_{3}\right)$, $2.57\left(\mathrm{~s}, 3 \mathrm{H}, \mathrm{CH}_{3}\right), 1.72\left(\mathrm{~s}, 6 \mathrm{H}, \mathrm{CH}_{3} \times 2\right) ;{ }^{13} \mathrm{C} \mathrm{NMR}(75$ $\left.\mathrm{MHz}, \mathrm{CDCl}_{3}\right) \delta: 162.47,158.64\left(\mathrm{~d},{ }^{1} J_{\mathrm{FC}}=240.8 \mathrm{~Hz}\right)$, $153.19,144.39,134.69\left(\mathrm{~d},{ }^{4} J_{\mathrm{FC}}=3.0 \mathrm{~Hz}\right), 120.79\left(\mathrm{~d},{ }^{3} J_{\mathrm{FC}}=\right.$ $7.5 \mathrm{~Hz}), 115.09\left(\mathrm{~d},{ }^{2} J_{\mathrm{FC}}=22.5 \mathrm{~Hz}\right), 112.67,71.22,35.69$, 28.95, 10.89. HR-ESI-MS cacld for $\mathrm{C}_{15} \mathrm{H}_{19} \mathrm{FN}_{3} \mathrm{O}_{2}[\mathrm{M}-\mathrm{H}]$ 290.1310 , found 290.1306 .

$\mathrm{N}$-(4-三氟甲基苯基)-3-(2-羟基丙基-2-基)-1,5-二甲 基- $1 H$-吡唑-4-甲酰胺(A3): 收率 49\%, 无色针状晶体. m.p. $231 \sim 233{ }^{\circ} \mathrm{C} ;{ }^{1} \mathrm{H}$ NMR $\left(300 \mathrm{MHz}, \mathrm{CDCl}_{3}\right) \delta: 10.63$ 
(s, 1H, NH), $7.62 \sim 7.58(\mathrm{~m}, 2 \mathrm{H}, \mathrm{ArH}), 7.03 \sim 6.98(\mathrm{~m}, 2 \mathrm{H}$, ArH), 3.72 (s, 3H, $\left.\mathrm{NCH}_{3}\right), 3.02$ (s, $\left.1 \mathrm{H}, \mathrm{OH}\right), 2.54$ (s, 3H, $\mathrm{CH}_{3}$ ), 1.70 (s, 6H, $\mathrm{CH}_{3} \times 2$ ); HR-ESI-MS cacld for $\mathrm{C}_{16} \mathrm{H}_{17} \mathrm{~F}_{3} \mathrm{~N}_{3} \mathrm{O}_{2}[\mathrm{M}-\mathrm{H}]^{-}$340.1278, found 340.1286.

$\mathrm{N}$-(2-氟苯基)-3-(1-差基环己基)-1,5-二甲基- $1 H$-吡 唑-4-甲酰胺(B1): 收率 52\%, 无色针状晶体. m.p. 189 $191{ }^{\circ} \mathrm{C} ;{ }^{1} \mathrm{H}$ NMR (300 MHz, $\left.\mathrm{CDCl}_{3}\right) \delta: 10.66(\mathrm{~s}, 1 \mathrm{H}, \mathrm{NH})$, $8.40 \sim 8.45$ (m, 1H, ArH), 6.95 7.21 (m, 3H, ArH), 3.75 (s, $\left.3 \mathrm{H}, \mathrm{NCH}_{3}\right), 3.14(\mathrm{~s}, 1 \mathrm{H}, \mathrm{OH}), 2.55\left(\mathrm{~s}, 3 \mathrm{H}, \mathrm{CH}_{3}\right), 2.08 \sim$ $1.61\left(\mathrm{~m}, 10 \mathrm{H}, \mathrm{CH}_{2} \times 5\right) ;{ }^{13} \mathrm{C} \mathrm{NMR}\left(75 \mathrm{MHz}, \mathrm{CDCl}_{3}\right) \delta$ : $162.92,153.98,152.81\left(\mathrm{~d},{ }^{1} J_{\mathrm{FC}}=243.0 \mathrm{~Hz}\right), 144.12$, $126.79\left(\mathrm{~d},{ }^{3} J_{\mathrm{FC}}=10.5 \mathrm{~Hz}\right), 123.74\left(\mathrm{~d},{ }^{2} J_{\mathrm{FC}}=34.5 \mathrm{~Hz}\right)$, $123.67\left(\mathrm{~d},{ }^{2} J_{\mathrm{FC}}=39.0 \mathrm{~Hz}\right), 122.13\left(\mathrm{~d},{ }^{4} J_{\mathrm{FC}}=1.5 \mathrm{~Hz}\right)$, $114.53\left(\mathrm{~d},{ }^{3} J_{\mathrm{FC}}=19.5 \mathrm{~Hz}\right), 112.61,71.58,36.38,35.78$, 25.11, 21.61, 11.03; HR-ESI-MS cacld for $\mathrm{C}_{18} \mathrm{H}_{21} \mathrm{FN}_{3} \mathrm{O}_{2}$ $[\mathrm{M}-\mathrm{H}]^{-}$330.1623, found 330.1628 .

$\mathrm{N}$-(4-氟苯基)-3-(1-差基环己基)-1,5-二甲基- $1 \mathrm{H}$-吡 唑-4-甲酰胺(B2): 收率 67\%, 无色针状晶体. m.p. 209 $210{ }^{\circ} \mathrm{C} ;{ }^{1} \mathrm{H}$ NMR (300 MHz, $\left.\mathrm{CDCl}_{3}\right) \delta: 10.79$ (s, $\left.1 \mathrm{H}, \mathrm{NH}\right)$, $7.61 \sim 7.57(\mathrm{~m}, 2 \mathrm{H}, \mathrm{ArH}), 7.02 \sim 6.96(\mathrm{~m}, 2 \mathrm{H}, \mathrm{ArH}), 3.72$ (s, 3H, $\left.\mathrm{NCH}_{3}\right), 2.99(\mathrm{~s}, 1 \mathrm{H}, \mathrm{OH}), 2.52\left(\mathrm{~s}, 3 \mathrm{H}, \mathrm{CH}_{3}\right), 2.04 \sim$ $1.60\left(\mathrm{~m}, 10 \mathrm{H}, \mathrm{CH}_{2} \times 5\right) ;{ }^{13} \mathrm{C}$ NMR $\left(75 \mathrm{MHz}, \mathrm{CDCl}_{3}\right) \delta$ : $162.59,158.54\left(\mathrm{~d},{ }^{1} J_{\mathrm{FC}}=240.8 \mathrm{~Hz}\right), 153.55,144.21$, $134.76\left(\mathrm{~d},{ }^{4} J_{\mathrm{FC}}=2.3 \mathrm{~Hz}\right), 120.67\left(\mathrm{~d},{ }^{3} J_{\mathrm{FC}}=7.5 \mathrm{~Hz}\right), 114.91$ $\left(\mathrm{d},{ }^{2} J_{\mathrm{FC}}=21.8 \mathrm{~Hz}\right), 112.76,72.03,36.30,35.70,25.13$, 21.59, 10.84. HR-ESI-MS cacld for $\mathrm{C}_{18} \mathrm{H}_{21} \mathrm{FN}_{3} \mathrm{O}_{2}[\mathrm{M}-\mathrm{H}]$ 330.1623 , found 330.1624 .

$N$-(4-三氟甲基苯基)-3-(1-羟基环已基)-1,5-二甲 基- $1 H$-吡唑-4-甲酰胺(B3): 收率 67\%, 无色针状晶体. m.p. $215 \sim 217{ }^{\circ} \mathrm{C} ;{ }^{1} \mathrm{H}$ NMR (300 MHz, $\mathrm{CDCl}_{3}$ ) $\delta: 11.12$ (s, $1 \mathrm{H}, \mathrm{NH}), 7.79 \sim 7.76(\mathrm{~m}, 2 \mathrm{H}, \mathrm{ArH}), 7.57 \sim 7.54(\mathrm{~m}, 2 \mathrm{H}$, $\mathrm{ArH}), 3.74$ (s, 3H, $\left.\mathrm{NCH}_{3}\right), 2.68(\mathrm{~s}, 1 \mathrm{H}, \mathrm{OH}), 2.55$ (s, 3H, $\left.\mathrm{CH}_{3}\right), 2.04 \sim 1.62\left(\mathrm{~m}, 10 \mathrm{H}, \mathrm{CH}_{2} \times 5\right) ;{ }^{13} \mathrm{C}$ NMR $(75 \mathrm{MHz}$, $\left.\mathrm{CDCl}_{3}\right) \delta: 162.76,153.29,144.81,141.90,125.85\left({ }^{4} J_{\mathrm{FC}}=\right.$ $3.8 \mathrm{~Hz}), 124.91,124.38,118.65,112.69,72.37,36.37$, 35.76, 25.07, 21.58, 10.88; HR-ESI-MS cacld for $\mathrm{C}_{19} \mathrm{H}_{21^{-}}$ $\mathrm{F}_{3} \mathrm{~N}_{3} \mathrm{O}_{2}[\mathrm{M}-\mathrm{H}]^{-}, 380.1591$, found 380.1587 .

\subsubsection{1,5-二甲基- $1 H$ - 吡唑-4-甲酰胺 $\mathbf{A 4} \sim \mathbf{A} 11$ 和 B4 $\sim$ B11 的合成}

以化合物 A4 的合成为例, 将吡唑-4-甲酸活化酯中 间体(I-9, $1.0 \mathrm{mmol}, 315 \mathrm{mg})$, 芐胺 $(1.5 \mathrm{mmol}, 161 \mathrm{mg}$ )和 THF $(30 \mathrm{~mL})$ 加入 $100 \mathrm{~mL}$ 单口圆底烧瓶中, 用油浴加热 至回流反应 $6 \mathrm{~h}, \mathrm{TLC}$ 监测反应完毕, 减压除去溶剂, 残 余物经硅胶柱层析[200 300 目硅胶, 以 $V$ (石油醚)： $V($ 乙酸乙酯 $)=3: 1$ 至 $1: 1$ 梯度]分离, 得到 $0.28 \mathrm{~g}$ 无色
晶体 A4, 收率 92\%. 采用同样的方法制得 $\mathbf{A 5} \sim \mathbf{A} 11$ 和 B4 $\sim$ B11.

$\mathrm{N}$-芐基-3-(2-羟基丙基-2-基)-1,5-二甲基- $1 \mathrm{H}$-吡唑-4甲酰胺(A4): 收率 $92 \%$, 白色固体. m.p. $152 \sim 154{ }^{\circ} \mathrm{C}$; ${ }^{1} \mathrm{H}$ NMR (300 MHz, $\left.\mathrm{CDCl}_{3}\right) \delta: 8.09$ (s, $\left.1 \mathrm{H}, \mathrm{NH}\right), 7.33 \sim$ $7.24(\mathrm{~m}, 5 \mathrm{H}, \mathrm{ArH}), 4.56\left(\mathrm{~d}, J=6.0 \mathrm{~Hz}, 2 \mathrm{H}, \mathrm{NCH}_{2}\right), 4.07$ (s, $1 \mathrm{H}, \mathrm{OH}), 3.70\left(\mathrm{~s}, 3 \mathrm{H}, \mathrm{NCH}_{3}\right), 2.45\left(\mathrm{~s}, 3 \mathrm{H}, \mathrm{CH}_{3}\right), 1.58$ (s, $\left.6 \mathrm{H}, \mathrm{CH}_{3} \times 2\right) ;{ }^{13} \mathrm{C}$ NMR $\left(75 \mathrm{MHz}, \mathrm{CDCl}_{3}\right) \delta: 164.89$, $154.90,142.08,138.20,128.26,127.47,126.92,112.20$, 70.14, 43.26, 35.70, 28.90, 10.96; IR (KBr) v: 3254, 3063, 2980, 2928, 1622, 1569, 1496, 1455, 1378, 1362, 1318, 1281, 1216, 1176, 951, 775, $697 \mathrm{~cm}^{-1}$; HR-ESI-MS cacld for $\mathrm{C}_{16} \mathrm{H}_{20} \mathrm{~N}_{3} \mathrm{O}_{2}[\mathrm{M}-\mathrm{H}]^{-}$286.1561, found 286.1556.

$\mathrm{N}$-(1-苯基乙基)-3-(2-羟基丙基-2-基)-1,5-二甲基$1 H$-吡唑-4-甲酰胺 (A5): 收率 89\%, 白色固体. m.p. $138 \sim 139{ }^{\circ} \mathrm{C} ;{ }^{1} \mathrm{H}$ NMR $\left(300 \mathrm{MHz}, \mathrm{CDCl}_{3}\right) \delta: 8.20(\mathrm{~s}, 1 \mathrm{H}$, $\mathrm{NH}), 7.38 \sim 7.21(\mathrm{~m}, 5 \mathrm{H}, \mathrm{ArH}), 5.31 \sim 5.21(\mathrm{~m}, 1 \mathrm{H}, \mathrm{CH})$, $3.90(\mathrm{~s}, 1 \mathrm{H}, \mathrm{OH}), 3.69\left(\mathrm{~s}, 3 \mathrm{H}, \mathrm{NCH}_{3}\right), 2.45\left(\mathrm{~s}, 3 \mathrm{H}, \mathrm{CH}_{3}\right)$, $1.62\left(\mathrm{~s}, 3 \mathrm{H}, \mathrm{CH}_{3}\right), 1.58\left(\mathrm{~s}, 3 \mathrm{H}, \mathrm{CH}_{3}\right), 1.54(\mathrm{~d}, J=6.0 \mathrm{~Hz}$, $\left.3 \mathrm{H}, \mathrm{CH}_{3}\right) ;{ }^{13} \mathrm{C}$ NMR $\left(75 \mathrm{MHz}, \mathrm{CDCl}_{3}\right) \delta: 163.92,154.61$, $143.36,142.25,128.21,126.75,125.91,112.42,70.24$, 48.59, 35.65, 28.94, 21.84, 10.90; HR-ESI-MS cacld for $\mathrm{C}_{17} \mathrm{H}_{22} \mathrm{~N}_{3} \mathrm{O}_{2}[\mathrm{M}-\mathrm{H}]^{-} \quad 300.1718$, found 300.1716 .

$\mathrm{N}$-(4-叔丁基苄基)-3-(2-羟基丙基-2-基)-1,5-二甲基1H-吡唑-4-甲酰胺(A6): 收率 85\%,白色固体. m.p. 182 $183{ }^{\circ} \mathrm{C} ;{ }^{1} \mathrm{H}$ NMR $\left(300 \mathrm{MHz}, \mathrm{CDCl}_{3}\right) \delta: 7.89(\mathrm{~s}, 1 \mathrm{H}, \mathrm{NH})$, $7.36 \sim 7.25(\mathrm{~m}, 4 \mathrm{H}, \mathrm{ArH}), 4.54\left(\mathrm{~d}, J=6.0 \mathrm{~Hz}, 2 \mathrm{H}, \mathrm{NCH}_{2}\right)$, $4.26(\mathrm{~s}, 1 \mathrm{H}, \mathrm{OH}), 3.70\left(\mathrm{~s}, 3 \mathrm{H}, \mathrm{CH}_{3}\right), 2.45\left(\mathrm{~s}, 3 \mathrm{H}, \mathrm{CH}_{3}\right)$, $1.59\left(\mathrm{~s}, 6 \mathrm{H}, \mathrm{CH}_{3}\right), 1.30\left(\mathrm{~s}, 9 \mathrm{H}, \mathrm{CH}_{3} \times 3\right) ;{ }^{13} \mathrm{C}$ NMR $(75$ $\left.\mathrm{MHz}, \mathrm{CDCl}_{3}\right) \delta: 164.97,155.15,149.88,141.75,135.07$, $127.14,125.18,112.26,70.02,42.95,35.71,34.14,31.00$, 28.90, 11.00; IR (KBr) v: 3246, 3064, 2980, 2927, 1620, 1567, 1495, 1454, 1425, 1377, 1361, 1317, 1280, 1215, $1176,951,733,698 \mathrm{~cm}^{-1}$; HR-ESI-MS cacld for $\mathrm{C}_{20} \mathrm{H}_{28^{-}}$ $\mathrm{N}_{3} \mathrm{O}_{2}[\mathrm{M}-\mathrm{H}]^{-}$342.2187, found 342.2182 .

$\mathrm{N}$-(2-氯苄基)-3-(2-羟基丙基-2-基)-1,5-二甲基- $1 \mathrm{H}$ 吡唑-4-甲酰胺(A7): 收率 85\%,白色固体. m.p. 162 $163{ }^{\circ} \mathrm{C} ;{ }^{1} \mathrm{H}$ NMR $\left(300 \mathrm{MHz}, \mathrm{CDCl}_{3}\right) \delta: 8.20(\mathrm{~s}, 1 \mathrm{H}, \mathrm{NH})$, $7.47 \sim 7.20$ (m, 4H, ArH), $4.65\left(\mathrm{~d}, J=6.0 \mathrm{~Hz}, 2 \mathrm{H}, \mathrm{NCH}_{2}\right)$, 4.16 (s, $1 \mathrm{H}, \mathrm{OH}), 3.71\left(\mathrm{~s}, 3 \mathrm{H}, \mathrm{NCH}_{3}\right), 2.47\left(\mathrm{~s}, 3 \mathrm{H}, \mathrm{CH}_{3}\right)$, $1.58\left(\mathrm{~s}, 6 \mathrm{H}, \mathrm{CH}_{3} \times 2\right) ;{ }^{13} \mathrm{C}$ NMR $\left(75 \mathrm{MHz}, \mathrm{CDCl}_{3}\right) \delta$ : $164.91,154.98,142.12,135.66,133.25,129.97,129.07$, 128.40, 126.69, 112.05, 70.11, 41.12, 35.71, 28.90, 10.97 . IR (KBr) v: 3245, 3066, 2980, 2930, 1621, 1566, 1474, 1443, 1377, 1362, 1315, 1279, 1215, 1176, 1053, 951, 753, 
$676 \mathrm{~cm}^{-1}$; HR-ESI-MS cacld for $\mathrm{C}_{16} \mathrm{H}_{19} \mathrm{ClN}_{3} \mathrm{O}_{2}[\mathrm{M}-\mathrm{H}]$ 320.1171 , found 320.1178 .

$\mathrm{N}$-(4-氟苠基)-3-(2-羟基丙基-2-基)-1,5-二甲基- $1 \mathrm{H}$ 吡唑-4-甲酰胺(A8): 收率 86\%, 白色固体. m.p. 133 $134{ }^{\circ} \mathrm{C} ;{ }^{1} \mathrm{H}$ NMR (300 MHz, $\left.\mathrm{CDCl}_{3}\right) \delta: 8.34$ (s, $\left.1 \mathrm{H}, \mathrm{NH}\right)$, $7.31 \sim 7.28(\mathrm{~m}, 2 \mathrm{H}, \mathrm{ArH}), 7.03 \sim 6.97$ (m, 2H, ArH), 4.52 $\left(\mathrm{d}, J=6.0 \mathrm{~Hz}, 2 \mathrm{H}, \mathrm{NCH}_{2}\right), 3.90(\mathrm{~s}, 1 \mathrm{H}, \mathrm{OH}), 3.71(\mathrm{~s}, 3 \mathrm{H}$, $\left.\mathrm{NCH}_{3}\right), 2.47\left(\mathrm{~s}, 3 \mathrm{H}, \mathrm{CH}_{3}\right), 1.59\left(\mathrm{~s}, 6 \mathrm{H}, \mathrm{CH}_{3} \times 2\right) ;{ }^{13} \mathrm{C} \mathrm{NMR}$ $\left(75 \mathrm{MHz}, \mathrm{CDCl}_{3}\right) \delta: 164.78,161.68\left(\mathrm{~d},{ }^{1} J_{\mathrm{FC}}=243.8 \mathrm{~Hz}\right)$, $154.57,142.47,134.15\left(\mathrm{~d},{ }^{4} J_{\mathrm{FC}}=3.0 \mathrm{~Hz}\right), 129.14\left(\mathrm{~d},{ }^{3} J_{\mathrm{FC}}=\right.$ $7.5 \mathrm{~Hz}), 115.03\left(\mathrm{~d},{ }^{2} J_{\mathrm{FC}}=21.0 \mathrm{~Hz}\right), 112.10,70.30,42.46$, 35.67, 28.90, 10.92; HR-ESI-MS cacld for $\mathrm{C}_{16} \mathrm{H}_{19} \mathrm{FN}_{3} \mathrm{O}_{2}$ $[\mathrm{M}-\mathrm{H}]^{-}$304.1467, found 304.1461.

$N$-叔丁基-3-(2-着基丙基-2-基)-1,5-二甲基- $1 H$-吡 唑-4-甲酰胺(A9): 收率 80\%,白色粉末固体. m.p. 171 $173{ }^{\circ} \mathrm{C} ;{ }^{1} \mathrm{H}$ NMR $\left(300 \mathrm{MHz}, \mathrm{CDCl}_{3}\right) \delta: 7.17$ (s, $\left.1 \mathrm{H}, \mathrm{NH}\right)$, $4.38(\mathrm{~s}, 1 \mathrm{H}, \mathrm{OH}), 3.70\left(\mathrm{~s}, 3 \mathrm{H}, \mathrm{NCH}_{3}\right), 2.43\left(\mathrm{~s}, 3 \mathrm{H}, \mathrm{CH}_{3}\right)$, $1.60\left(\mathrm{~s}, 6 \mathrm{H}, \mathrm{CH}_{3} \times 2\right), 1.42\left(\mathrm{~s}, 9 \mathrm{H}, \mathrm{CH}_{3} \times 3\right) ;{ }^{13} \mathrm{C} \mathrm{NMR}(75$ $\left.\mathrm{MHz}, \mathrm{CDCl}_{3}\right) \delta: 164.73,155.01,140.76,113.86,69.82$, 50.99, 35.66, 28.79, 28.51, 10.81; HR-ESI-MS cacld for $\mathrm{C}_{13} \mathrm{H}_{22} \mathrm{~N}_{3} \mathrm{O}_{2}[\mathrm{M}-\mathrm{H}]^{-}$252.1718, found 252.1711.

$\mathrm{N}$-(噻唑-2-基)-3-(2-羟基丙基-2-基)-1,5-二甲基- $1 H$ 吡唑-4-甲酰胺(A10): 收率 79\%, 浅黄色固体. m.p. 167 $168{ }^{\circ} \mathrm{C} ;{ }^{1} \mathrm{H}$ NMR $\left(300 \mathrm{MHz}, \mathrm{CDCl}_{3}\right) \delta: 7.34$ (d, $J=$ $3.0 \mathrm{~Hz}, 1 \mathrm{H}$, Thiazole-H), 6.88 (d, $J=3.0 \mathrm{~Hz}, 1 \mathrm{H}$, Thiazole$\mathrm{H}), 3.73\left(\mathrm{~s}, 3 \mathrm{H}, \mathrm{NCH}_{3}\right), 2.57\left(\mathrm{~s}, 3 \mathrm{H}, \mathrm{CH}_{3}\right), 1.69(\mathrm{~s}, 6 \mathrm{H}$, $\mathrm{CH}_{3} \times 2$ ); HR-ESI-MS cacld for $\mathrm{C}_{12} \mathrm{H}_{15} \mathrm{SN}_{4} \mathrm{O}_{2}[\mathrm{M}-\mathrm{H}]^{-}$ 279.0921, found 279.0918.

$N$-(1,3,4-噻二唑-2-基)-3-(2-羟基丙基-2-基)-1,5-二 甲基-1H-吡唑-4-甲酰胺(A11): 收率 75\%, 浅黄色固体. m.p. 202 203 ${ }^{\circ} \mathrm{C} ;{ }^{1} \mathrm{H}$ NMR $\left(300 \mathrm{MHz}, \mathrm{CDCl}_{3}\right) \delta$ : 8.80 (s, $1 \mathrm{H}$, Thiadiazole-H), 3.78 (s, 3H, $\left.\mathrm{NCH}_{3}\right), 2.61$ (s, 3H, $\left.\mathrm{CH}_{3}\right)$, $1.75\left(\mathrm{~s}, 6 \mathrm{H}, \mathrm{CH}_{3} \times 2\right) ;{ }^{13} \mathrm{C} \mathrm{NMR}\left(75 \mathrm{MHz}, \mathrm{CDCl}_{3}\right) \delta$ : $161.53,154.30,150.96,146.81,146.22,109.82,71.35$, 35.82, 29.41, 11.15; HR-ESI-MS cacld for $\mathrm{C}_{11} \mathrm{H}_{14} \mathrm{SN}_{5} \mathrm{O}_{2}$ $[\mathrm{M}-\mathrm{H}]^{-}$280.0874, found 280.0876 .

$\mathrm{N}$-苠基-3-(1-着基环己基)-1,5-二甲基- $1 H$-吡唑-4-甲 酰胺(B4): 收率 $72 \%$, 白色固体. m.p. $167 \sim 169{ }^{\circ} \mathrm{C} ;{ }^{1} \mathrm{H}$ NMR (300 MHz, $\left.\mathrm{CDCl}_{3}\right) \delta: 8.35(\mathrm{~s}, 1 \mathrm{H}, \mathrm{NH}), 7.33 \sim 7.28$ (m, 5H, ArH) , 4.54 (d, $\left.J=6.0 \mathrm{~Hz}, 2 \mathrm{H}, \mathrm{NCH}_{2}\right), 3.70$ (s, 3H, $\left.\mathrm{NCH}_{3}\right), 3.43(\mathrm{~s}, 1 \mathrm{H}, \mathrm{OH}), 2.45\left(\mathrm{~s}, 3 \mathrm{H}, \mathrm{CH}_{3}\right), 2.03 \sim 1.56$ $\left(\mathrm{m}, 10 \mathrm{H}, \mathrm{CH}_{2} \times 5\right) ;{ }^{13} \mathrm{C} \mathrm{NMR}\left(75 \mathrm{MHz}, \mathrm{CDCl}_{3}\right) \delta: 164.82$, $154.81,142.28,138.23,128.22,127.57,126.87,112.42$, $71.16,43.25,36.26,35.70,25.20,21.50,10.85$. HR-ESIMS cacld for $\mathrm{C}_{19} \mathrm{H}_{24} \mathrm{~N}_{3} \mathrm{O}_{2}[\mathrm{M}-\mathrm{H}]^{-}$326.1874, found
326.1876 .

$N$-(1-苯基乙基)-3-(1-差基环己基)-1,5-二甲基- $1 H$ 吡唑-4-甲酰胺(B5): 收率 75\%, 白色固体. m.p. 175 $177{ }^{\circ} \mathrm{C} ;{ }^{1} \mathrm{H}$ NMR (300 MHz, $\left.\mathrm{CDCl}_{3}\right) \delta: 8.45$ (s, 1H, NH), $7.39 \sim 7.21(\mathrm{~m}, 5 \mathrm{H}, \mathrm{ArH}), 5.30 \sim 5.21(\mathrm{~m}, 1 \mathrm{H}, \mathrm{CH}), 3.70$ $\left(\mathrm{s}, 3 \mathrm{H}, \mathrm{NCH}_{3}\right), 3.14(\mathrm{~s}, 1 \mathrm{H}, \mathrm{OH}), 2.46\left(\mathrm{~s}, 3 \mathrm{H}, \mathrm{CH}_{3}\right), 2.04 \sim$ $1.52\left(\mathrm{~m}, 13 \mathrm{H}, \mathrm{CH}_{2} \times 5+\mathrm{CH}_{3}\right) ;{ }^{13} \mathrm{C} \mathrm{NMR}(75 \mathrm{MHz}$, $\left.\mathrm{CDCl}_{3}\right) \delta: 163.80,154.47,143.43,142.48,128.18,126.70$, $125.96,112.68,71.33,48.48,36.24,35.67,25.20,21.86$, 21.48 ; 10.81. HR-ESI-MS cacld for $\mathrm{C}_{20} \mathrm{H}_{26} \mathrm{~N}_{3} \mathrm{O}_{2}[\mathrm{M}-\mathrm{H}]$ 340.2031 , found 340.2034 .

$N$-(4-叔丁基苄基)-3-(1-羟基环己基)-1,5-二甲基1H-吡唑-4-甲酰胺(B6): 收率 76\%，白色固体. m.p. 222 $223{ }^{\circ} \mathrm{C} ;{ }^{1} \mathrm{H}$ NMR (300 MHz, $\left.\mathrm{CDCl}_{3}\right) \delta: 8.04(\mathrm{~s}, 1 \mathrm{H}$, $\mathrm{NH}), 7.37 \sim 7.26(\mathrm{~m}, 4 \mathrm{H}, \mathrm{ArH}), 4.55(\mathrm{~d}, J=6.0 \mathrm{~Hz}, 2 \mathrm{H}$, $\left.\mathrm{NCH}_{2}\right), 3.72\left(\mathrm{~s}, 3 \mathrm{H}, \mathrm{CH}_{3}\right), 3.37(\mathrm{~s}, 1 \mathrm{H}, \mathrm{OH}), 2.47(\mathrm{~s}, 3 \mathrm{H}$, $\left.\mathrm{CH}_{3}\right), 2.05 \sim 1.51\left(\mathrm{~m}, 10 \mathrm{H}, \mathrm{CH}_{2} \times 5\right), 1.31\left(\mathrm{~s}, 9 \mathrm{H}, \mathrm{CH}_{3} \times\right.$ $3) ;{ }^{13} \mathrm{C} \mathrm{NMR}\left(75 \mathrm{MHz}, \mathrm{CDCl}_{3}\right) \delta: 164.84,154.95,149.86$, $141.93,135.13,127.27,125.17,112.55,71.14,42.93$, $36.29,35.73,34.14,31.00,25.22,21.53,10.89$; HR-ESIMS cacld for $\mathrm{C}_{23} \mathrm{H}_{32} \mathrm{~N}_{3} \mathrm{O}_{2}[\mathrm{M}-\mathrm{H}]^{-} 382.2500$, found 382.2502 .

$N$-(2-氯苄基)-3-(1-羟基环己基)-1,5-二甲基- $1 H$-吡 唑-4-甲酰胺(B7): 收率 $82 \%$, 白色固体. m.p. 144 $145{ }^{\circ} \mathrm{C} ;{ }^{1} \mathrm{H}$ NMR $\left(300 \mathrm{MHz}, \mathrm{CDCl}_{3}\right) \delta: 8.41(\mathrm{t}, J=5.8 \mathrm{~Hz}$, $1 \mathrm{H}, \mathrm{NH}), 7.48 \sim 7.36(\mathrm{~m}, 2 \mathrm{H}, \mathrm{ArH}), 7.24 \sim 7.21(\mathrm{~m}, 2 \mathrm{H}$, $\mathrm{ArH}), 4.65\left(\mathrm{~d}, J=5.8 \mathrm{~Hz}, 2 \mathrm{H}, \mathrm{NCH}_{2}\right), 3.72\left(\mathrm{~s}, 3 \mathrm{H}, \mathrm{NCH}_{3}\right)$, $3.42(\mathrm{~s}, 1 \mathrm{H}, \mathrm{OH}), 2.48\left(\mathrm{~s}, 3 \mathrm{H}, \mathrm{CH}_{3}\right), 2.00 \sim 1.51(\mathrm{~m}, 10 \mathrm{H}$, $\left.\mathrm{CH}_{2} \times 5\right) ;{ }^{13} \mathrm{C} \mathrm{NMR}\left(75 \mathrm{MHz}, \mathrm{CDCl}_{3}\right) \delta: 164.85,154.84$, $142.30,135.74,133.31,130.09$, 129.07, 128.37, 126.67, $112.30,71.16,41.04,36.27,35.72,25.19,21.52,10.88$; IR (KBr) v: 3245, 3063, 2930, 2854, 1622, 1559, 1473, 1444, $1386,1312,1275,1255,1189,1054,962,750 \mathrm{~cm}^{-1}$; HR-ESI-MS cacld for $\mathrm{C}_{19} \mathrm{H}_{23} \mathrm{ClN}_{3} \mathrm{O}_{2}[\mathrm{M}-\mathrm{H}]^{-}$360.1484, found 360.1488 .

$N$-(4-氟苠基)-3-(1-羟基环己基)-1,5-二甲基- $1 H$-吡 唑-4-甲酰胺(B8)：收率 86\%，白色固体. m.p. 189 $190{ }^{\circ} \mathrm{C} ;{ }^{1} \mathrm{H}$ NMR $\left(300 \mathrm{MHz}, \mathrm{CDCl}_{3}\right) \delta: 8.53$ (s, $\left.1 \mathrm{H}, \mathrm{NH}\right)$, 7.30 (dd, $J=8.5,5.5 \mathrm{~Hz}, 2 \mathrm{H}, \mathrm{ArH}), 6.99$ (t, $J=8.7 \mathrm{~Hz}, 2 \mathrm{H}$, ArH), 4.50 (d, $\left.J=5.8 \mathrm{~Hz}, 2 \mathrm{H}, \mathrm{NCH}_{2}\right), 3.70\left(\mathrm{~s}, 3 \mathrm{H}, \mathrm{NCH}_{3}\right)$, $3.21(\mathrm{~s}, 1 \mathrm{H}, \mathrm{OH}), 2.46\left(\mathrm{~s}, 3 \mathrm{H}, \mathrm{CH}_{3}\right), 2.00 \sim 1.56(\mathrm{~m}, 10 \mathrm{H}$, $\left.\mathrm{CH}_{2} \times 5\right) ;{ }^{13} \mathrm{C} \mathrm{NMR}\left(75 \mathrm{MHz}, \mathrm{CDCl}_{3}\right) \delta: 164.73,161.68$ $\left(\mathrm{d},{ }^{1} J_{\mathrm{FC}}=243.8 \mathrm{~Hz}\right), 154.53,142.61,134.15\left(\mathrm{~d},{ }^{4} J_{\mathrm{FC}}=3.0\right.$ $\mathrm{Hz}), 129.19\left(\mathrm{~d},{ }^{3} J_{\mathrm{FC}}=8.3 \mathrm{~Hz}\right), 114.98\left(\mathrm{~d},{ }^{2} J_{\mathrm{FC}}=21.8 \mathrm{~Hz}\right)$, $112.33,71.31,42.44,36.24,35.68,25.15,21.47,10.81$; IR 
(KBr) v: 3245, 3066, 2930, 2854, 1621, 1569, 1508, 1435, 1417, 1384, 1315, 1279, 1254, 1220, 1156, 962, $830 \mathrm{~cm}^{-1}$; HR-ESI-MS cacld for $\mathrm{C}_{19} \mathrm{H}_{23} \mathrm{FN}_{3} \mathrm{O}_{2}[\mathrm{M}-\mathrm{H}]^{-} 344.1780$, found 344.1781 .

$N$-叔丁基-3-(1-着基环已基)-1,5-二甲基- $1 H$-吡唑-4甲酰胺(B9): 收率 76\%, 白色粉末固体. m.p. 168 $170{ }^{\circ} \mathrm{C} ;{ }^{1} \mathrm{H}$ NMR $\left(300 \mathrm{MHz}, \mathrm{CDCl}_{3}\right) \delta: 7.48$ (s, 1H, NH), 3.71 (s, 3H, $\left.\mathrm{NCH}_{3}\right), 3.66(\mathrm{~s}, 1 \mathrm{H}, \mathrm{OH}), 2.44\left(\mathrm{~s}, 3 \mathrm{H}, \mathrm{CH}_{3}\right)$, 2.03-1.57 (m, $\left.10 \mathrm{H}, \mathrm{CH}_{2} \times 5\right), 1.42\left(\mathrm{~s}, 9 \mathrm{H}, \mathrm{CH}_{3} \times 3\right) ;{ }^{13} \mathrm{C}$ NMR (75 MHz, $\left.\mathrm{CDCl}_{3}\right) \delta: 164.64,154.86,141.07,114.02$, 70.98, 50.86, 36.34, 35.67, 28.54, 25.30, 21.61, 10.75; IR (KBr) v: 3255, 3078, 2931, 2856, 1628, 1575, 1507, 1455, 1388, 1363, 1327, 1275, 1254, 1225, 1155, 1058, 963, 908, $736 \mathrm{~cm}^{-1}$; HR-ESI-MS cacld for $\mathrm{C}_{16} \mathrm{H}_{26} \mathrm{~N}_{3} \mathrm{O}_{2}[\mathrm{M}-\mathrm{H}]$ 292.2031, found 292.2035.

$N$-(噻唑-2-基)-3-(1-差基环己基)-1,5-二甲基- $1 H$-吡 唑-4-甲酰胺(B10): 收率 79\%, 浅黄色固体. m.p. 181 $183{ }^{\circ} \mathrm{C} .{ }^{1} \mathrm{H}$ NMR $\left(300 \mathrm{MHz}, \mathrm{CDCl}_{3}\right) \delta: 13.68$ (s, 1H, NH), 7.26 (d, $J=3.6 \mathrm{~Hz}, 1 \mathrm{H}$, Thiazole-H) , 6.83 (d, $J=3.6 \mathrm{~Hz}$, $1 \mathrm{H}$, Thiazole-H), $6.24(\mathrm{~s}, 1 \mathrm{H}, \mathrm{OH}), 3.74\left(\mathrm{~s}, 3 \mathrm{H}, \mathrm{NCH}_{3}\right)$, $2.55\left(\mathrm{~s}, 3 \mathrm{H}, \mathrm{CH}_{3}\right), 2.04 \sim 1.59\left(\mathrm{~m}, 10 \mathrm{H}, \mathrm{CH}_{2} \times 5\right) ;{ }^{13} \mathrm{C}$ NMR (75 MHz, $\left.\mathrm{CDCl}_{3}\right) \delta: 161.82,159.25,155.27,145.46$, 136.35, 112.03, 110.38, 71.05, 36.51, 35.67, 25.28, 21.33, 11.02; HR-ESI-MS cacld for $\mathrm{C}_{15} \mathrm{H}_{19} \mathrm{SN}_{4} \mathrm{O}_{2}[\mathrm{M}-\mathrm{H}]$ 319.1234, found 319.1236 .

$N$-(1,3,4-噻二唑-2-基)-3-(1-羟基环己基)-1,5-二甲 基-1H-吡唑-4-甲酰胺(B11): 收率 78\%, 浅黄色固体. m.p. $201 \sim 203{ }^{\circ} \mathrm{C} ;{ }^{1} \mathrm{H}$ NMR (300 MHz, $\left.\mathrm{CDCl}_{3}\right) \delta: 14.03$ (s, 1H, NH), 8.73 (s, 1H, Thiadiazole-H), 5.58 (s, 1H, OH), $3.75\left(\mathrm{~s}, 3 \mathrm{H}, \mathrm{NCH}_{3}\right), 2.56\left(\mathrm{~s}, 3 \mathrm{H}, \mathrm{CH}_{3}\right), 2.19 \sim 1.60(\mathrm{~m}$, $\left.10 \mathrm{H}, \mathrm{CH}_{2} \times 5\right) ;{ }^{13} \mathrm{C} \mathrm{NMR}\left(75 \mathrm{MHz}, \mathrm{CDCl}_{3}\right) \delta: 161.74$, $159.27,155.43,146.63,145.92,109.82,71.74,36.38$, 35.81, 25.13, 21.30, 11.09; HR-ESI-MS cacld for $\mathrm{C}_{14} \mathrm{H}_{18^{-}}$ $\mathrm{SN}_{5} \mathrm{O}_{2}[\mathrm{M}-\mathrm{H}]^{-}$320.1187, found 320.1184 .

\section{3 化合物 $A 7$ 的晶体 $X$ 射线衍射}

将高纯度的 $20 \mathrm{mg}$ 化合物 $\mathbf{A 7}$ 放入 $5 \mathrm{~mL}$ 透明螺口 玻璃小瓶中, 用 $3 \mathrm{~mL}$ 乙醇/正己烷体系(体积比 $1: 5$ )溶 解, 专用保鲜膜封口并在保鲜膜上部扎 $2 \sim 3$ 个针孔, 保 持阴凉使溶剂以一定速度自然缓慢挥发, 大约 7 $8 \mathrm{~d}$ 后 析出无色透明片状晶体. 该化合物晶体结构参数: 晶体 大小 $0.54 \mathrm{~nm} \times 0.40 \mathrm{~nm} \times 0.30 \mathrm{~mm}$, 分子式 $\mathrm{C}_{16} \mathrm{H}_{20} \mathrm{Cl}-$ $\mathrm{N}_{3} \mathrm{O}_{2}, M_{\mathrm{r}}=321.80$, monoclinic, $a=1.34076(6) \mathrm{nm}, b=$ $0.64230(2) \mathrm{nm}, c=1.86252(8) \mathrm{nm}, \beta=98.475(5)^{\circ}, V=$ $1.58644(11) \mathrm{nm}^{3}, \rho=1.347 \mathrm{mg} / \mathrm{mm}^{3}$, 空间群 $P 2_{1} / n, Z=$ $4, \mu($ Mo K $\alpha)=0.252 \mathrm{~mm}^{-1}, F(000)=680, S=1.043$. 在 $T$
$=105.6 \mathrm{~K}$ 温度下共收集到 7016 个衍射点, 其中 3105 个独立衍射点 $\left(R_{\mathrm{int}}=0.0217\right)$ 用于结构解析和精修, 最终 结构因子 $R_{1}=0.0516, w R_{2}=0.1069$ (all data). 化合物 $\mathbf{A 7}$ 的所有参数和结构信息已保存在剑桥晶体结构数据中 心(CDC ID 1923299). 这些数据可以免费从剑桥晶体结 构数据中心获得.

\section{4 化合物 $A$ 和 $B$ 的杀菌活性}

所用植物病原菌小麦赤霉菌 (Fusarium graminearum)、瓜果腐霉菌(Pythium aphanidermatum)、番茄灰 霉菌(Botrytis cinerea)、辣椒疫霉菌(Phytophthora capsici)、水稻纹枯菌(Thanatephorus cucumeris)和水稻疫霉菌 (Phytophthora infestans) 由中国农业大学植物保护学院 植物病理系分离并保存, 活性测试时需要进一步活化. 化合物 $\mathbf{A}$ 和 $\mathbf{B}$ 的离体杀菌活性测试按照前文采用菌丝 生长速率法 ${ }^{[18 ~ 20]}$ 进行测定.

辅助材料(Supporting Information) 化合物 $\mathbf{A 1} \sim \mathbf{A 1 1}$, B1 B11 的 ${ }^{1} \mathrm{H}$ NMR 和 ${ }^{13} \mathrm{C} \mathrm{NMR}$ 图谱以及化合物 $\mathbf{A} 7$ 的 晶体结构参数. 这些材料可以免费从本刊网站 (http://sioc-journal.cn/)上下载.

\section{References}

[1] Zhang, Y.; Chen, Z.; Nie, J.; Zhang, F. G.; Ma, J. A. J. Org. Chem. 2019, 84, 7148.

[2] Zhang, A.; Yue, Y.; Yang, J.; Shi, J.; Tao, K.; Jin, H.; Hou, T. J. Agric. Food Chem. 2019, 67, 5008.

[3] Zhang, A.; Zhou, J.; Tao, K.; Hou, T.; Jin, H. Bioorg. Med. Chem. Lett. 2018, 28, 3042.

[4] Walter, H.; Lamberth, C.; Corsi, C. Monatsh. Chem. 2018, 149, 791.

[5] Yan, Z.; Liu, A.; Huang, M.; Liu, M.; Pei, H.; Huang, L.; Yi, H.; Liu, W.; Hu, A. Eur. J. Med. Chem. 2018, 149, 170.

[6] Mu, J. X.; Shi, Y. X.; Yang, M. Y.; Sun, Z. H.; Liu, X. H.; Li, B. J.; Sun, N. B. Molecules 2016, 21, 68.

[7] Du, S.; Tian, Z.; Yang, D.; Li, X.; Li, H.; Jia, C.; Che, C.; Wang, M.; Qin, Z. Molecules 2015, 20, 8395.

[8] Sun, J.; Zhou, Y. Molecules 2015, 20, 4383.

[9] Wu, J.; Wang, J.; Hu, D.; He, M.; Jin, L.; Song, B. Chem. Central J. 2012, 6, 51 .

[10] Yang, Y.; Jiang, J. Z.; Qimei, L. B.; Yan, X. J.; Zhao, J. X.; Yuan, H. Z.; Qin, Z. H.; Wang, M. A. Molecule 2010, 15, 7075.

[11] Zhao, J.; Dong, H. B.; Yang, M. Y.; Du, J.; Jiang, J. Z.; Wang, M. A. J. Asian Nat. Prod. Res. 2014, 16, 312.

[12] Maneerat, W.; Phakhodee, W.; Ritthiwigrom, T.; Cheenpracha, S.; Promgool, T.; Yossathera, K.; Laphookhieo, S. Fitoterapia 2012, 83,1110

[13] Hitesh, V.; Motwani, M. D. R.; Luke, R.; Odell, A. H.; Mats, L. Eur. J. Med. Chem. 2015, 90, 462.

[14] Hernandez-Altamirano, J. M.; Largo-Gosens, A.; Martinez-Rubio, R.; Pereda, D.; Alvarez, J. M.; Acebes, J. L.; Encina, A.; Garcia-Angulo, P. Planta 2018, 247, 987.

[15] Li, X.; Han, X.; He, M.; Xiao, Y.; Qin, Z. Bioorg. Med. Chem. Lett. 2017, 26, 5936.

[16] Zhao, Y.; Tang, B.; Liu, X. L.; Li, W. Z.; Huang, M. Y.; Wang, M. A. Chin. J. Org. Chem. 2017, 37, 975 (in Chinese). (赵宇, 汤博, 刘釒䂞, 李婉祯, 黄铭一, 王明安, 有机化学, 2017, 37, 975.) 
[17] Zhao, Y.; Guan, A. Y.; Li, W. Z.; Wang, W. W.; Liu, X. L.; Wang, M. A. Chin. J. Org. Chem. 2019, 39, 1344 (in Chinese).

(赵宇, 关爱莹, 李婉祯, 王卫伟, 刘金否, 王明安, 有机化学, 2019, 39, 1344.)

[18] Zhao, Y.; Tang, B.; Guan, A. Y.; Wang, W. W.; Zhang, Z. H.; Wang, M. A. Synthesis 2017, 49, 4663.
[19] Tang, B.; Guan, A. Y.; Zhao, Y.; Jiang, J. Z.; Wang, M. A.; Zhou, L. G. Chin. J. Chem. 2017, 35, 1133 .

[20] Liu, B.; Han, J. T.; Tang, B.; Wang, M. A. Chin. J. Org. Chem. 2014, 34, 1442 (in Chinese).

(刘斌，韩金涛，汤博，王明安，有机化学, 2014, 34, 1442.)

(Zhao, C.) 